\title{
Gastrojejunocolic fistula: A case report
}

Khairussaleh BJ and Kyaw TH

Department of Surgery, International Islamic University Malaysia, Pahang.

\section{ABSTRACT}

Benign gastrojejunocolic fistula is mostly due to Bilroth II operations. It typically presents with a triad of feculent vomiting, weight loss and chronic diarrhoea but sometimes the diagnosis is not straight forward. We discuss a case that presented and was investigated as intestinal obstruction however diagnosed with gastrojejunocolic fistula during surgery.

KEYWORDS: Gastrojejunocolic fistula, Bilroth II

\section{INTRODUCTION}

Gastrojejunocolic fistula (GJCF) can be benign or malignant. Most of the malignant fistulas are due tumour infiltration or tumour recurrence. Benign GJCF is rare nowadays due to a reduction in peptic ulcer operations. However, clinicians should be alert to this condition especially in patients with previous history of Bilroth II operation.

\section{CASE REPORT}

A 53 year-old gentleman presented with a one-week episode of post-prandial feculent projectile vomiting, associated with generalized abdominal pain and distension. He was able to pass flatus but has had no bowel motion during admission. He also complained of passing loose stools for four months prior to the admission. There were constitutional symptoms of loosing weight and appetite. He had undergone gastrojejunostomy and truncal vagotomy for gastric outlet obstruction in 1999. On examination he was cachexic, peripherally oedematous with a tinge of jaundice. The abdomen was distended, mildly tender with ascites and hyperactive bowel sounds.

His blood investigation showed severe malnutrition; he was anaemic with hemoglobin of $6.2 \mathrm{~g} / \mathrm{dl}$, hypokalaemic with potassium of $2.7 \mathrm{mmol} / \mathrm{l}$, hypoalbuminaemic with albumin $15 \mathrm{~g} / \mathrm{l}$ and several documented episodes of hypoglycaemia whilst in the ward. Abdominal radiography showed dilated large bowels (Figure 1).

Corresponding author;

Dr Khairussaleh Bin Jalaludin,

Department of Surgery,

Kulliyah of Medicine,

International Islamic University Malaysia,

Jalan Hospital, 25170 Kuantan, Pahang

Email: krssaleh@yahoo.com

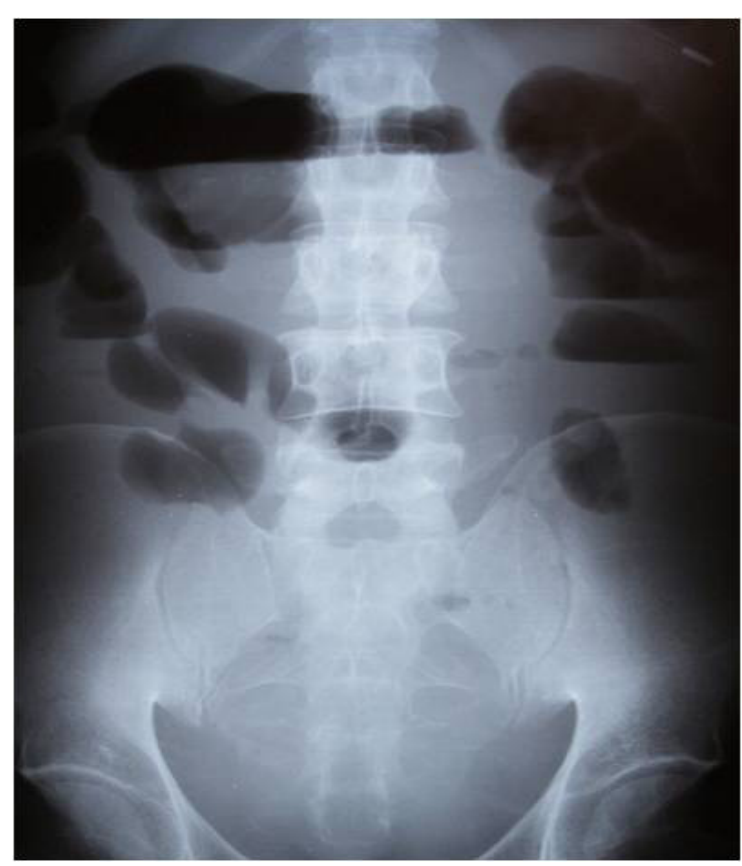

Figure 1. Plain radiographic x-ray of the abdomen revealing dilated large bowels

He had upper and lower endoscopy performed. Oesophagogastroduodenoscopy (OGDS) showed stomach filled with food particles. Colonoscopy was normal but the physician was unable to advance the scope beyond the splenic flexure. CT scan showed dilated stomach and small bowels, and mildly dilated ascending colon (Figure 2). The contrast study does not reveal any fistula. There was no abnormal mass noted.

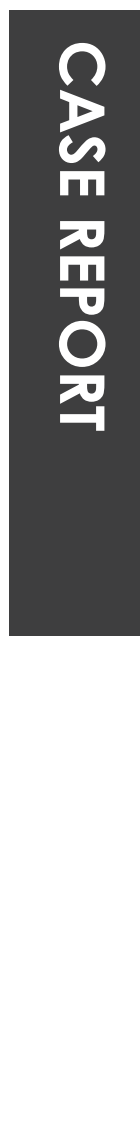




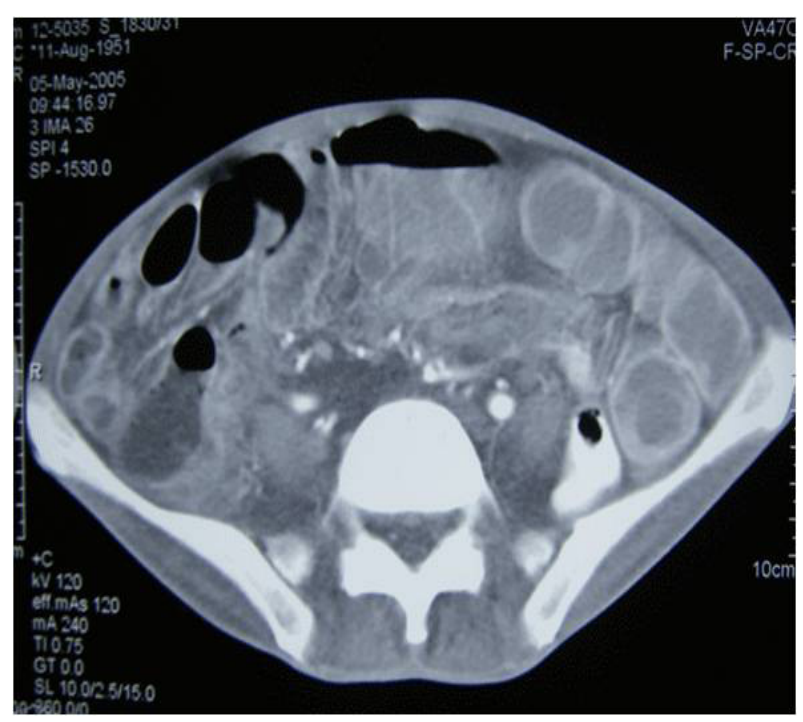

Figure 2. CT abdomen showing dilated bowels

With provisional diagnosis of obstruction secondary to adhesion, laparotomy was performed. There were $1500 \mathrm{mls}$ of ascitic fluid. Stomach and small bowel were dilated. The stomach, jejunum and transverse colon were locked at one point. Upon further dissection, gastrojejunocolic fistula was revealed. This patient had a short retrocolic proximal limb to gastrojejunostomy done in the previous surgery. There was a stomal ulcer and histopathology showed a chronic ulcer. Staged procedure was planned on table. Fistula at the stomach and jejunum were closed primarily (Figure 3 ). The colonic fistula was brought out as loop colostomy. New gastrojejunostomy was created.

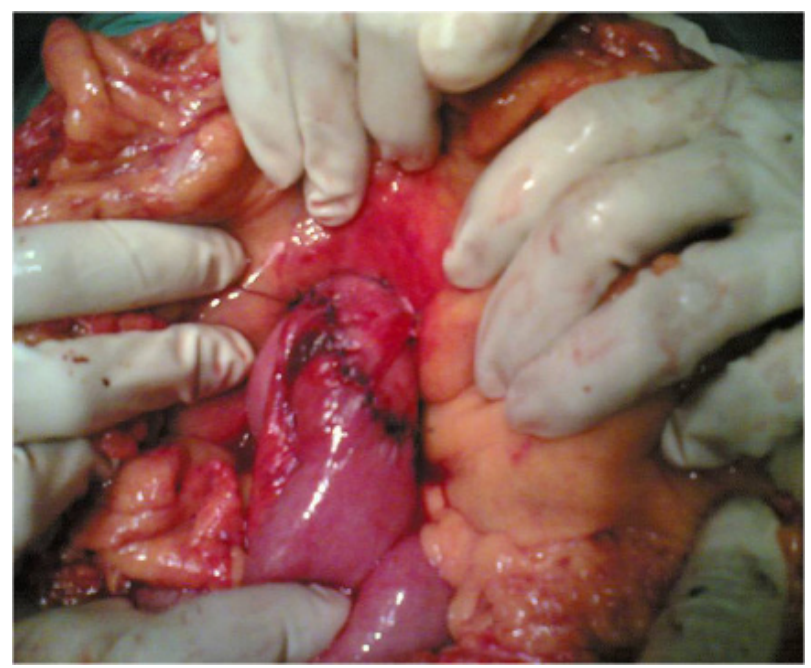

Figure 3: Gastrojejunal repair

Post operation, he was started on total parenteral nutrition (TPN) and other supportive measures. Unfortunately, he never recovered and succumbed to septicaemic shock and died day eight postoperatively.

\section{DISCUSSION}

Gastrojejunocolic fistula (GJCF) was first reported by Czerny in 1903 and there were many cases reported in the early part of last century. ${ }^{1,2}$ Fortunately it is a rare occurrence in the twenty-first century mostly due to effective peptic ulcer treatment. It is almost exclusively a Bilroth II complication although Bilroth I can also cause this fistula. It can present as early as few months or as late as thirty years or more..$^{2-4}$ It is thought to be due to incomplete operative procedure that results in a stomal ulcer and this ulcer triggers inflammation and erosion into the nearby colon, thereby creating a fistula. Another common feature is a short proximal jejunal limb to the gastojejunostomy during the first operation.

Diagnosing gastrojunocolic fistula is not always straight forward as illustrated in this case. GJCF should be suspected in patients who had Bilroth II operations especially those operated for peptic ulcer disease. It usually present as a triad of feculent vomiting or breath, weight loss, and chronic diarrhea. ${ }^{5}$ Laboratory investigation will reveal a certain degree of malnourishment and dehydration. The severity will depend on the size of the fistula as well as the duration of the illness. Radioimaging and endoscopy will normally confirm the diagnosis in a majority of cases. Unfortunately in this case, OGDS was unsatisfactory due to excessive food and fecal material, colonoscopy was incomplete and the $\mathrm{CT}$ with barium enema was not diagnostic. ${ }^{1,5}$

Most fistulas are diagnosed pre-operatively. Preoperative optimization is crucial for these patients as a majority of them are malnourished. Mortality following surgical repair has been reported to be as high as $40 \%$ especially for single stage operations. Staged operations are one of the important management steps in most cases especially for those diagnosed during laparotomy. Staged repair of GJCF is considered as damage-control surgery and requires preliminary diversion colostomy. Combination of proper preoperative optimization and staged operation has reduced the mortality to $5 \% .^{7}$ In selected cases proper pre-operative optimization and nutrition will also allow a single stage operation. ${ }^{2,6}$

\section{Acknowledgements:}

We wish to extend our gratitude to all staff in Department of Surgery, HTAA and IIUM for their support and assistance. 


\section{REFERENCES}

1. Subramaniasivam N, Ananthakrishnan N, Kate V, Smile SR, Jagdish S, Srinivasan K. Gastrojejunocolic fistula following surgery for peptic ulcer. Trop Gastroenterol 1997; 18:183-7

2. Jiang Z, Zhang S, Li Y,et al. Diagnosis and treatment of gastrojejunocolic fistula and nutrition. World J Gastroenterol 1998; 4(Suppl 2):86-8

3. Ohta M, Konno H, Tanaka T, et al. Gastrojejunocolic fistula after gastrectomy with Billroth II reconstruction: report of a case. Surg Today 2002; 32:367-70

4. Johnston DW, Walker JH, Scardaccione P. Management of Gastrojejunocolic fistula. Med Serv J Can 1966; 22:807-12

5. Marshall SF, Knud-Hansen J. Gastrojejunocolic and gastrocolic fistulas. Ann Surg 1957; 145:770-82

6. Rahman M, Miah MA, Sarwar JM, Rahman M. Gastrojejunocolic fistula following surgery for peptic ulcer complications: two case reports. Malaysian Med J 2002; 11:125-8

7. Lowdon AGR: Gastrojejunocolic fistula. Br J Surg 1953; 41:113-28 
